Supporting Information

for

\title{
Light Mediated Properties of a Thiolato-derivative of Vitamin $\mathbf{B}_{12}$
}

\author{
Megan J. Toda, ${ }^{1}$ Piotr Lodowski, ${ }^{2}$ Todd Thurman, ${ }^{1}$ and Pawel M. Kozlowski ${ }^{1 *}$ \\ ${ }^{1}$ Department of Chemistry, University of Louisville, Louisville, Kentucky 40292, United States \\ ${ }^{2}$ Department of Theoretical Chemistry, Institute of Chemistry, University of Silesia in Katowice, \\ Szkolna 9, PL-40 006 Katowice, Poland
}

*Phone: (502) 852-6609. Fax: (502) 852-8149. E-mail: pawel@louisville.edu 


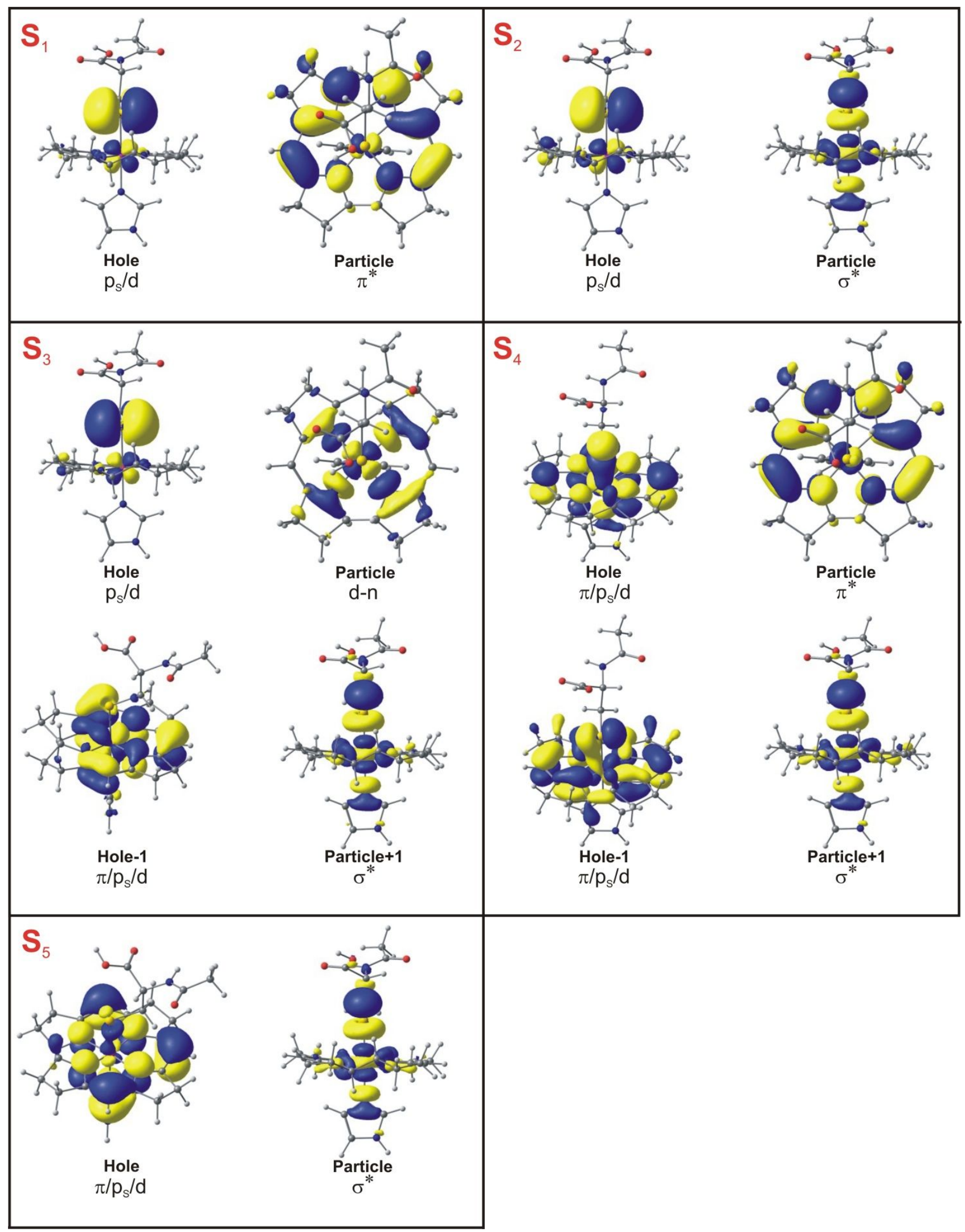

Figure S1. Natural transition orbitals (NTO) describing singlet, lowest, excited electronic states $\mathrm{S}_{1}-\mathrm{S}_{5}$. 


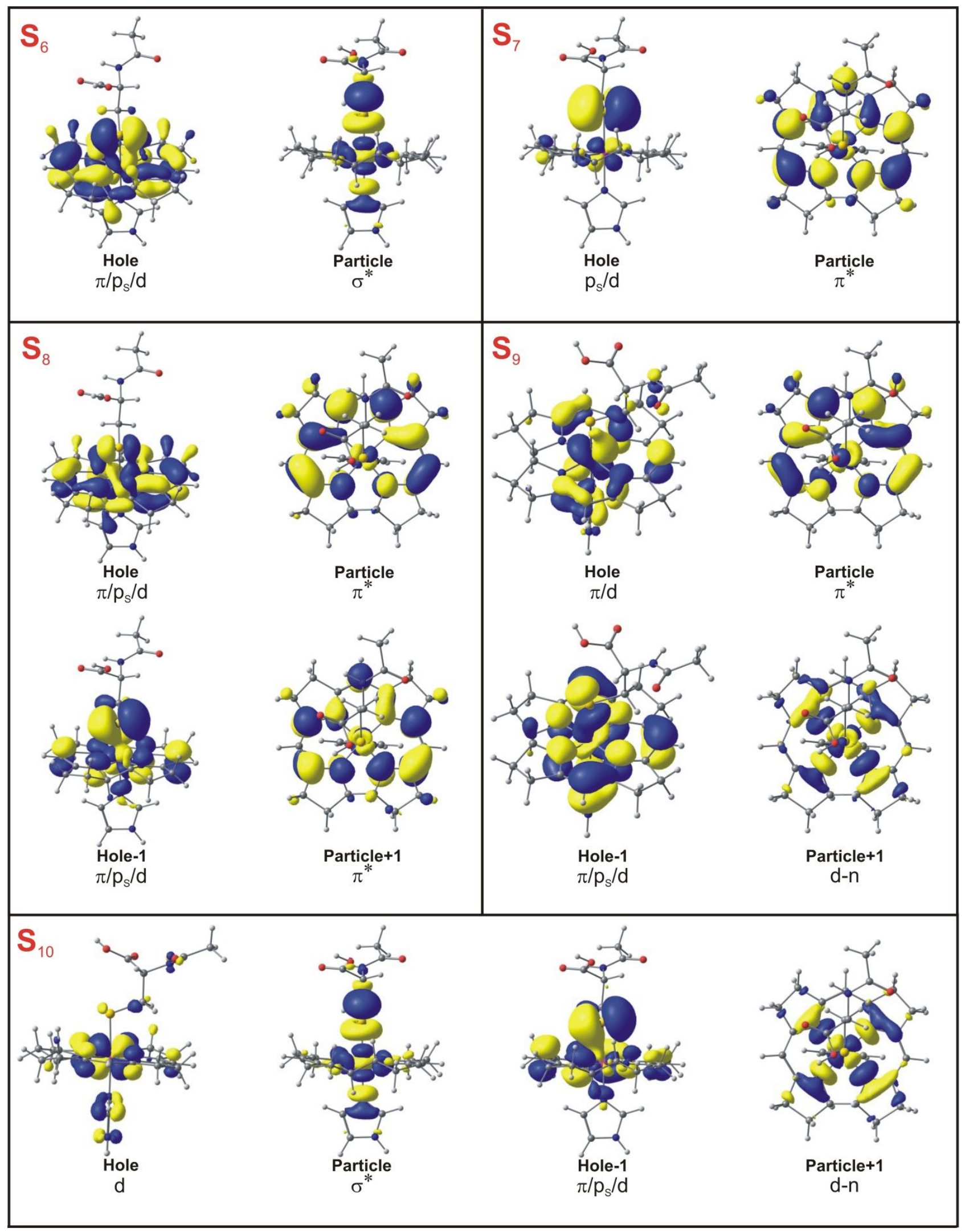

Figure S2. Natural transition orbitals (NTO) describing singlet, lowest, excited electronic states $\mathrm{S}_{6}-\mathrm{S}_{10}$. 


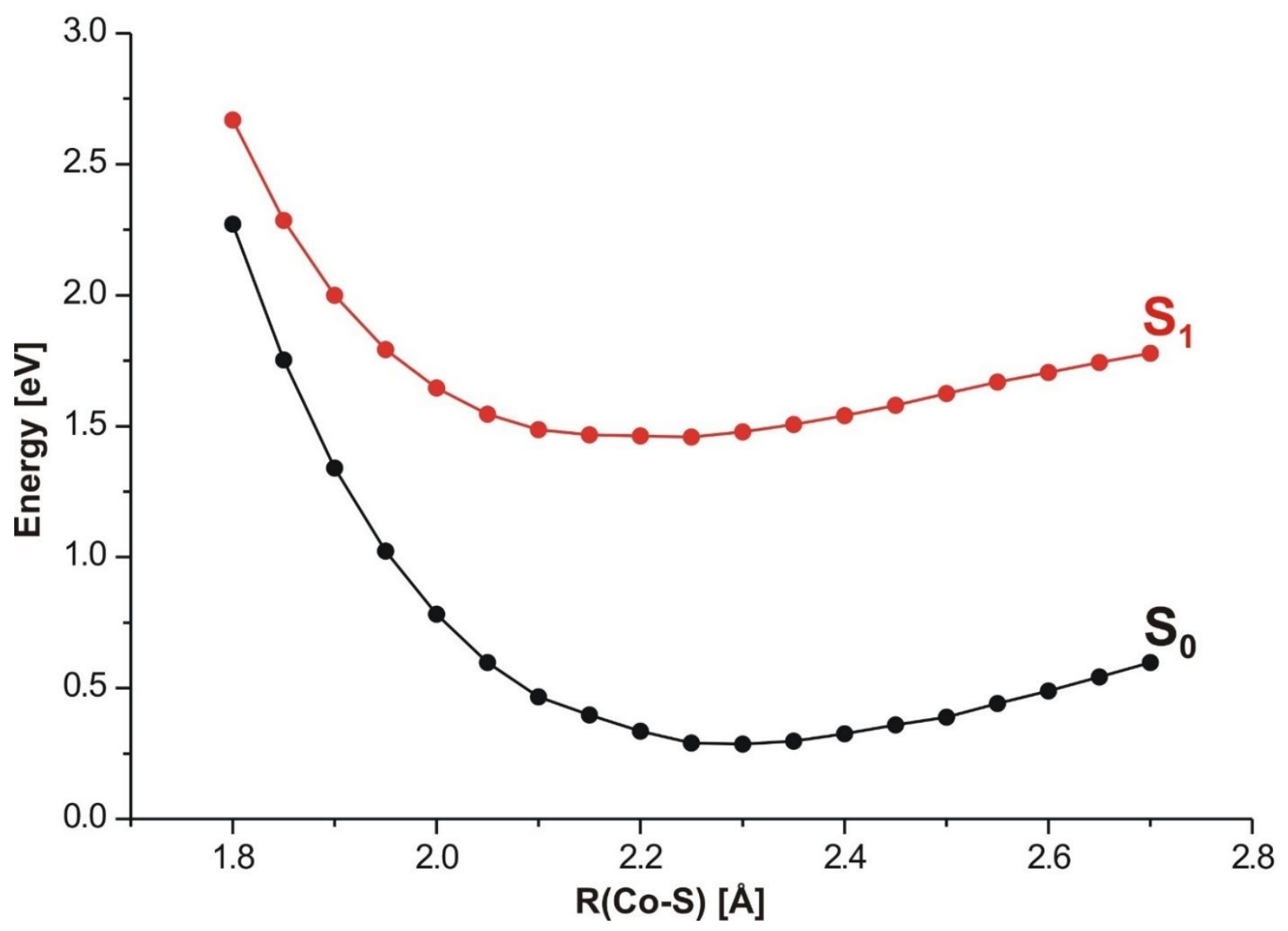

Figure S3. Potential energy curves of the $\mathrm{S}_{0}$ ground and $\mathrm{S}_{1}$ excited states as function of Co-S axial bond length for optimized geometry $\mathrm{S}_{1}$ state of $\mathrm{Im}-\left[\mathrm{Co}^{\mathrm{III}}(\mathrm{corrin})\right]-\mathrm{NAC}^{+}$model complex. 


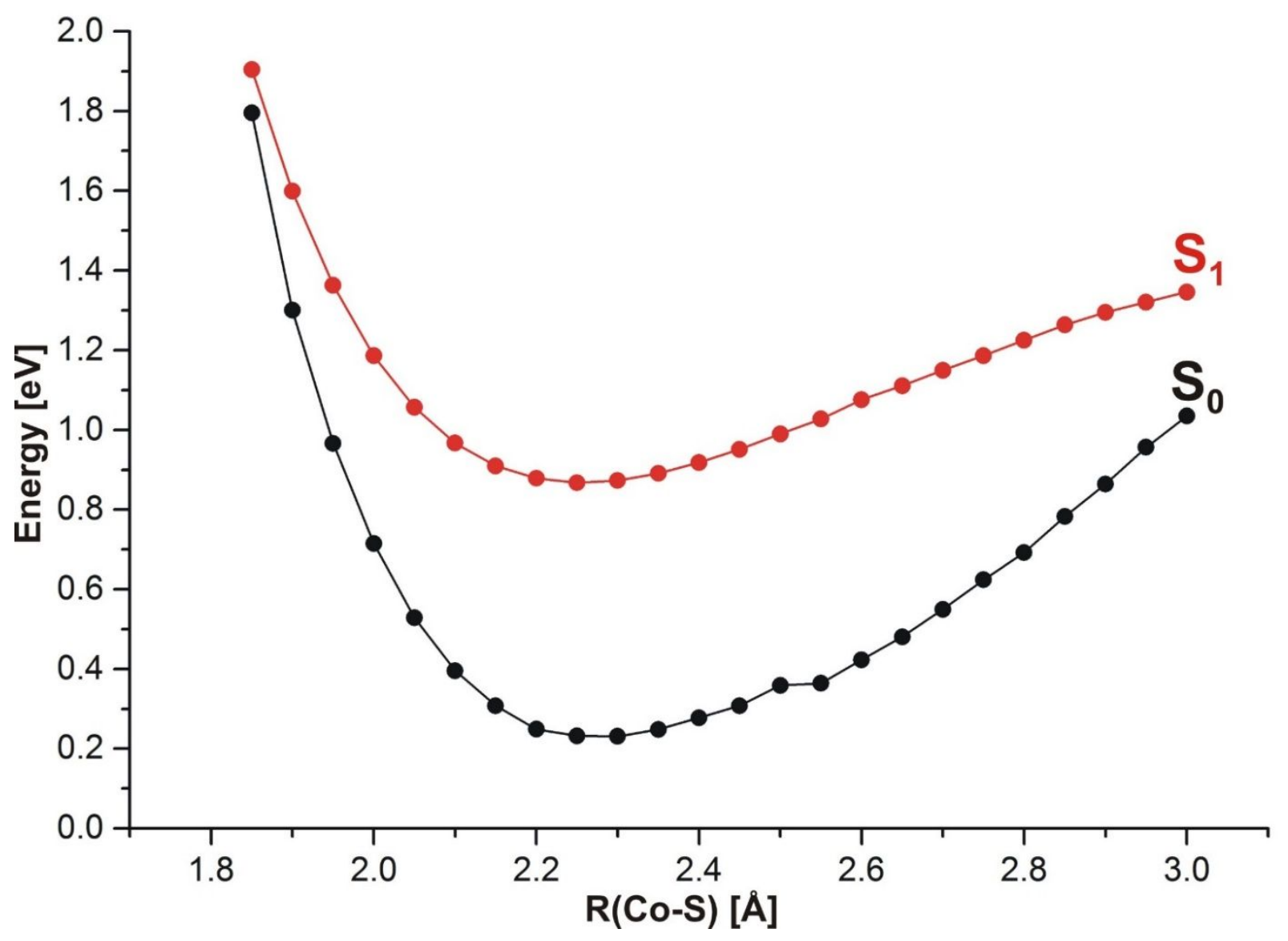

Figure S4. Potential energy curves of the $\mathrm{S}_{0}$ ground and $\mathrm{S}_{1}$ excited states as function of Co-S axial bond length for optimized geometry $\mathrm{S}_{1}$ state of $\left[\mathrm{Co}^{\mathrm{III}}\right.$ (corrin)]-NAC ${ }^{+}$model complex. 

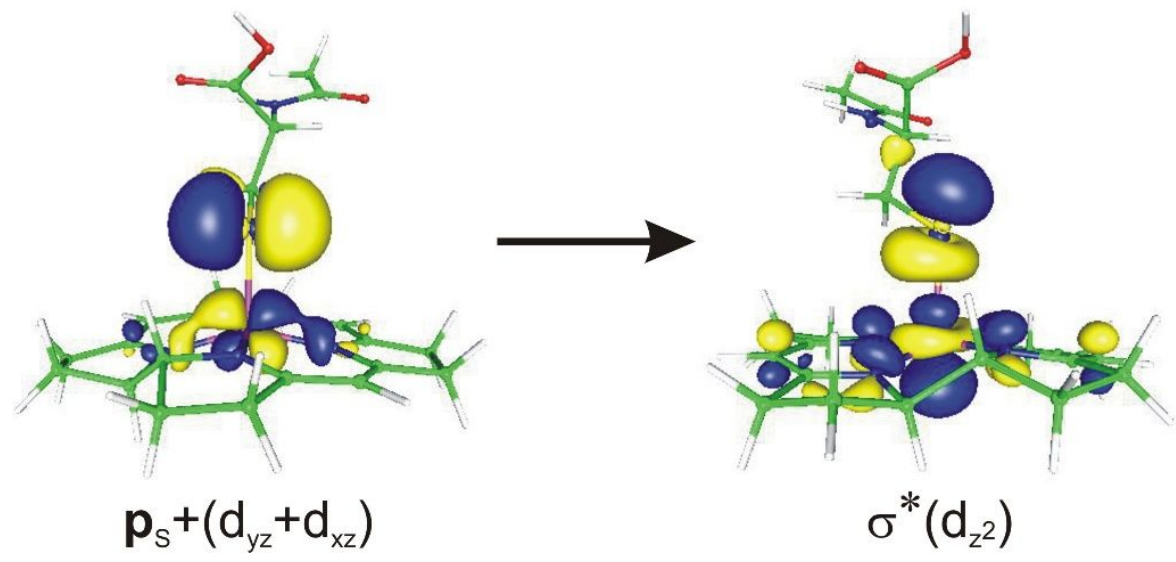

Figure S5. Kohn-Sham orbitals involved in electronic excitation at minimum energy on $\mathrm{S}_{1}$ PEC of $\left[\mathrm{Co}^{\mathrm{III}}\right.$ (corrin) $]-\mathrm{NAC}^{+}$model complex (Figure S4). 


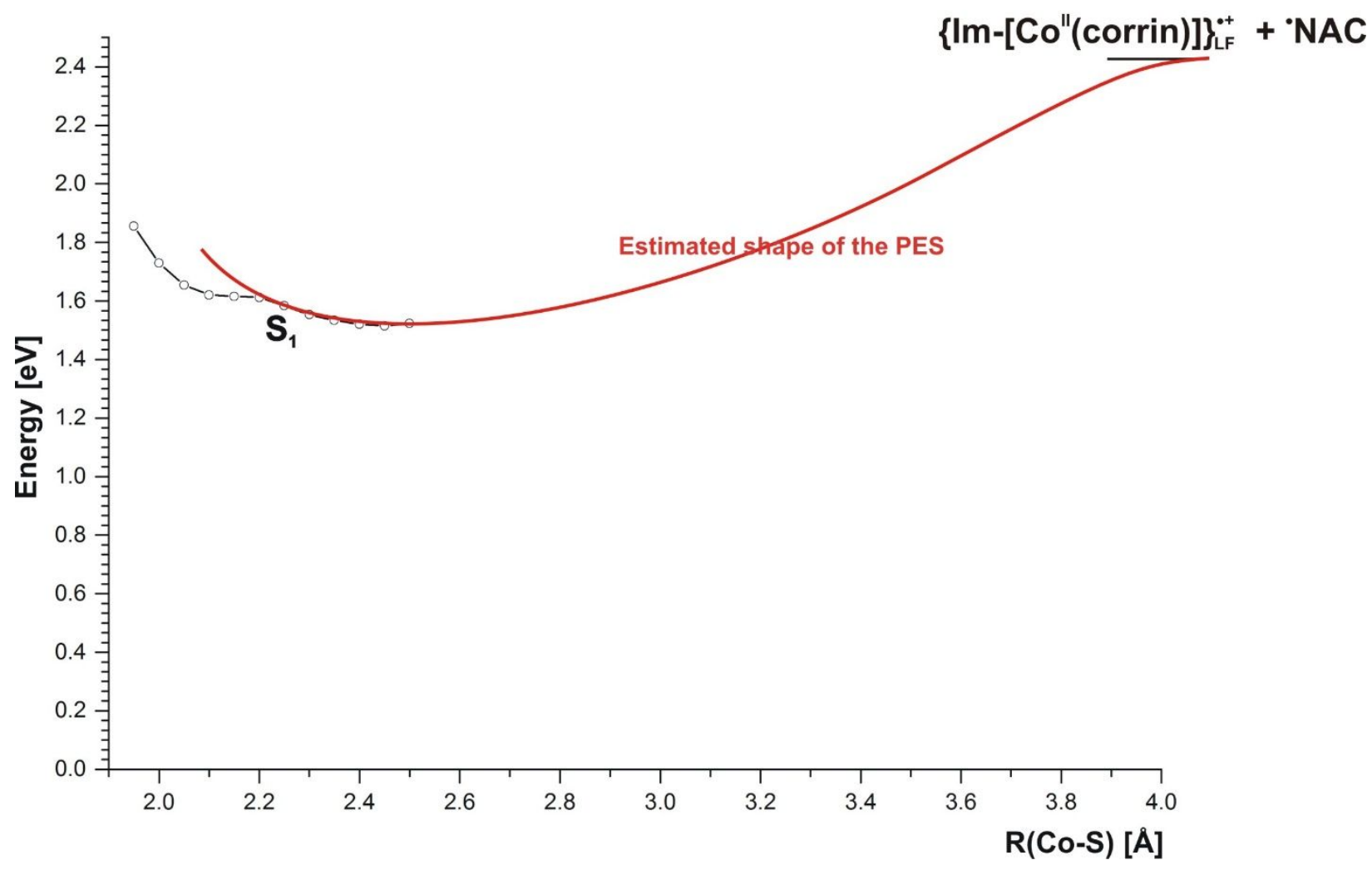

Figure S6. The estimation of the PES beyond $2.5 \AA$ based on a fragment of the $\mathrm{S}_{1}$ PES along the Co-S bond for a constant Co- $\mathrm{N}_{\mathrm{Im}}=2.3 \AA$ distance. 
Table S1. Forty five, vertical singlet states for the $\mathrm{Im}-\left[\mathrm{Co}{ }^{\mathrm{III}}(\right.$ corrin $\left.)\right]-\mathrm{NAC}^{+}$model complex.

\begin{tabular}{|c|c|c|c|c|c|c|c|}
\hline & $\mathrm{E}[\mathrm{eV}]$ & $\lambda[\mathrm{nm}]$ & $f$ & $\%$ & Character & & $\begin{array}{l}\text { Exp. } \lambda[\mathrm{nm}] \\
(\mathrm{eV})^{\mathrm{b})}\end{array}$ \\
\hline $\mathrm{S}_{1}$ & 1.70 & 729.6 & 0.0012 & 95 & $\mathrm{H} \rightarrow \mathrm{L}$ & $\mathrm{p}_{\mathrm{S}}+\mathrm{d}_{\mathrm{yz}} \rightarrow \pi^{*}$ & $615 \mathrm{sh}$ \\
\hline $\mathrm{S}_{2}$ & 1.85 & 669.9 & 0.0002 & 96 & $\mathrm{H} \rightarrow \mathrm{L}+1$ & $\mathrm{p}_{\mathrm{s}}+\mathrm{d}_{\mathrm{yz}} \rightarrow \sigma^{*}\left(\mathrm{~d}_{\mathrm{z}}^{2}\right)$ & $(2.02)$ \\
\hline \multirow[t]{2}{*}{$\mathrm{S}_{3}$} & 2.30 & 538.1 & 0.0067 & $\begin{array}{l}57 \\
26\end{array}$ & $\begin{array}{l}\mathrm{H} \rightarrow \mathrm{L}+2 \\
\mathrm{H}-1 \rightarrow \mathrm{L}+1\end{array}$ & $\begin{array}{l}\mathrm{p}_{\mathrm{S}}+\mathrm{d}_{\mathrm{yz}} \rightarrow \mathrm{d}_{\mathrm{xz}}-\mathrm{n} \\
\pi+\left(\mathrm{d}_{\mathrm{z}}^{2} / \mathrm{p}_{\mathrm{s}}\right) \rightarrow \sigma^{*}\left(\mathrm{~d}_{\mathrm{z}}^{2}\right)\end{array}$ & \\
\hline & & & & 12 & $\mathrm{H}-1 \rightarrow \mathrm{L}$ & $\pi+\left(\mathrm{d}_{\mathrm{z}}^{2} / \mathrm{p}_{\mathrm{S}}\right) \rightarrow \pi^{*}$ & \\
\hline \multirow[t]{2}{*}{$\mathrm{S}_{4}$} & 2.34 & 529.5 & 0.0180 & 43 & $\mathrm{H}-1 \rightarrow \mathrm{L}$ & $\pi+\left(\mathrm{d}_{\mathrm{z}}^{2} / \mathrm{p}_{\mathrm{S}}\right) \rightarrow \pi^{*}$ & 561 \\
\hline & & & & $\begin{array}{l}32 \\
15\end{array}$ & $\begin{array}{l}\mathrm{H} \rightarrow \mathrm{L}+2 \\
\mathrm{H}-1 \rightarrow \mathrm{L}+1\end{array}$ & $\begin{array}{l}\mathrm{p}_{\mathrm{s}}+\mathrm{d}_{\mathrm{yz}} \rightarrow \mathrm{d}_{\mathrm{xz}}-\mathrm{n} \\
\pi+\left(\mathrm{d}_{\mathrm{z}}^{2} / \mathrm{p}_{\mathrm{s}}\right) \rightarrow \sigma^{*}\left(\mathrm{~d}_{\mathrm{z}}^{2}\right)\end{array}$ & $(2.21)$ \\
\hline \multirow[t]{2}{*}{$\mathrm{S}_{5}$} & 2.38 & 521.4 & 0.0207 & 49 & $\mathrm{H}-1 \rightarrow \mathrm{L}+1$ & $\pi+\left(\mathrm{d}_{\mathrm{z}}^{2} / \mathrm{p}_{\mathrm{s}}\right) \rightarrow \sigma^{*}\left(\mathrm{~d}_{\mathrm{z}}^{2}\right)$ & \\
\hline & & & & 27 & $\mathrm{H}-1 \rightarrow \mathrm{L}$ & $\pi+\left(\mathrm{d}_{\mathrm{z}}^{2} / \mathrm{p}_{\mathrm{S}}\right) \rightarrow \pi^{*}$ & \\
\hline $\mathrm{S}_{6}$ & 2.47 & 502.3 & 0.0052 & 78 & $\mathrm{H}-2 \rightarrow \mathrm{L}+1$ & $\pi+\left(\mathrm{d}_{\mathrm{yz}} / \mathrm{p}_{\mathrm{s}}\right) \rightarrow \sigma^{*}\left(\mathrm{~d}_{\mathrm{z}}^{2}\right)$ & \\
\hline $\mathrm{S}_{7}$ & 2.51 & 493.7 & 0.0004 & 97 & $\mathrm{H} \rightarrow \mathrm{L}+3$ & $\mathrm{p}_{\mathrm{S}}+\mathrm{d}_{\mathrm{yz}} \rightarrow \pi^{*}$ & \\
\hline \multirow[t]{2}{*}{$\mathrm{S}_{8}$} & 2.64 & 470.4 & 0.0216 & 69 & $\mathrm{H}-2 \rightarrow \mathrm{L}$ & $\pi+\left(\mathrm{d}_{\mathrm{yz}} / \mathrm{p}_{\mathrm{S}}\right) \rightarrow \pi^{*}$ & \\
\hline & & & & 11 & $\mathrm{H}-1 \rightarrow \mathrm{L}+3$ & $\pi+\left(\mathrm{d}_{\mathrm{z}}^{2} / \mathrm{p}_{\mathrm{s}}\right) \rightarrow \pi^{*}$ & 534 \\
\hline \multirow[t]{2}{*}{$\mathrm{S}_{9}$} & 2.71 & 458.0 & 0.0233 & 69 & $\mathrm{H}-3 \rightarrow \mathrm{L}$ & $d_{x z} \rightarrow \pi^{*}$ & $(2.32)$ \\
\hline & & & & 20 & $\mathrm{H}-1 \rightarrow \mathrm{L}+2$ & $\pi+\left(\mathrm{d}_{\mathrm{z}}^{2} / \mathrm{p}_{\mathrm{s}}\right) \rightarrow \mathrm{d}_{\mathrm{xz}}-\mathrm{n}$ & \\
\hline \multirow[t]{2}{*}{$\mathrm{S}_{10}$} & 2.77 & 446.9 & 0.0022 & 78 & $\mathrm{H}-3 \rightarrow \mathrm{L}+1$ & $d_{x z} \rightarrow \sigma^{*}\left(d_{z}^{2}\right)$ & \\
\hline & & & & 10 & $\mathrm{H}-2 \rightarrow \mathrm{L}+2$ & $\pi+\left(\mathrm{d}_{\mathrm{yz}} / \mathrm{p}_{\mathrm{s}}\right) \rightarrow \mathrm{d}_{\mathrm{xz}}-\mathrm{n}$ & \\
\hline $\mathrm{S}_{11}$ & 2.79 & 444.1 & 0.0139 & $\begin{array}{l}67 \\
16\end{array}$ & $\begin{array}{l}\mathrm{H}-1 \rightarrow \mathrm{L}+2 \\
\mathrm{H}-3 \rightarrow \mathrm{L}\end{array}$ & $\begin{array}{l}\pi+\left(\mathrm{d}_{\mathrm{z}}^{2} / \mathrm{p}_{\mathrm{s}}\right) \rightarrow \mathrm{d}_{\mathrm{xz}}-\mathrm{n} \\
\mathrm{d}_{\mathrm{x}} \rightarrow \pi^{*}\end{array}$ & $\begin{array}{l}498 \\
(2.49)\end{array}$ \\
\hline $\mathrm{S}_{12}$ & 2.85 & 435.6 & 0.0039 & 91 & $\mathrm{H}-4 \rightarrow \mathrm{L}$ & $\mathrm{d}_{\mathrm{x} z}^{2}-\mathrm{y}^{2} \rightarrow \pi^{*}$ & \\
\hline $\mathrm{S}_{13}$ & 3.06 & 404.8 & 0.0006 & 82 & $\mathrm{H}-2 \rightarrow \mathrm{L}+2$ & $\pi+\left(\mathrm{d}_{\mathrm{yz}} / \mathrm{p}_{\mathrm{s}}\right) \rightarrow \mathrm{d}_{\mathrm{xz}}-\mathrm{n}$ & \\
\hline $\mathrm{S}_{14}$ & 3.12 & 397.7 & 0.0003 & 100 & $\mathrm{H}-5 \rightarrow \mathrm{L}$ & $\mathrm{p}_{\mathrm{O}(\mathrm{NAC})} \rightarrow \pi^{*}$ & \\
\hline $\mathrm{S}_{15}$ & 3.18 & 390.3 & 0.0006 & 94 & $\mathrm{H}-6 \rightarrow \mathrm{L}$ & $\pi_{\mathrm{Im}} \rightarrow \pi^{*}$ & 433 \\
\hline $\mathrm{S}_{16}$ & 3.18 & 389.8 & 0.0082 & 98 & $\mathrm{H} \rightarrow \mathrm{L}+4$ & $\mathrm{p}_{\mathrm{s}}+\mathrm{d}_{\mathrm{yz}} \rightarrow \pi_{\mathrm{NAC}}^{*}$ & $(2.86)$ \\
\hline $\mathrm{S}_{17}$ & 3.19 & 388.7 & 0.0005 & 93 & $\mathrm{H} \rightarrow \mathrm{L}+5$ & $\mathrm{p}_{\mathrm{S}}+\mathrm{d}_{\mathrm{yz}} \rightarrow \pi^{*}$ & \\
\hline $\mathrm{S}_{18}$ & 3.20 & 388.1 & 0.0019 & 96 & $\mathrm{H}-5 \rightarrow \mathrm{L}+1$ & $\mathrm{p}_{\mathrm{O}(\mathrm{NAC})} \rightarrow \sigma^{*}\left(\mathrm{~d}_{\mathrm{z}}^{2}\right)$ & \\
\hline $\mathrm{S}_{19}$ & 3.25 & 381.2 & 0.0001 & 94 & $\mathrm{H}-6 \rightarrow \mathrm{L}+1$ & $\pi_{\mathrm{Im}} \rightarrow \sigma^{*}\left(\mathrm{~d}_{\mathrm{z}}^{2}\right)$ & \\
\hline \multirow[t]{3}{*}{$\mathrm{S}_{20}$} & 3.29 & 376.6 & 0.0398 & 51 & $\mathrm{H}-1 \rightarrow \mathrm{L}+3$ & $\pi+\left(\mathrm{d}_{\mathrm{z}}^{2} / \mathrm{p}_{\mathrm{s}}\right) \rightarrow \pi^{*}$ & \\
\hline & & & & 12 & $\mathrm{H}-4 \rightarrow \mathrm{L}+1$ & $\mathrm{~d}_{\mathrm{x}}^{2}-\mathrm{y}^{2} \rightarrow \sigma^{*}\left(\mathrm{~d}_{\mathrm{z}}^{2}\right)$ & \\
\hline & & & & 12 & $\mathrm{H}-8 \rightarrow \mathrm{L}$ & $\left(\mathrm{d}_{\mathrm{yz}} / \mathrm{p}_{\mathrm{S}}\right)+\pi \rightarrow \pi^{*}$ & \\
\hline$S_{21}$ & 3.32 & 373.2 & 0.0101 & 66 & $\mathrm{H}-2 \rightarrow \mathrm{L}+3$ & $\pi+\left(\mathrm{d}_{\mathrm{yz}} / \mathrm{p}_{\mathrm{s}}\right) \rightarrow \pi^{*}$ & \\
\hline \multirow[t]{3}{*}{$\mathrm{S}_{22}$} & 3.36 & 368.7 & 0.0277 & 40 & $\mathrm{H}-4 \rightarrow \mathrm{L}+1$ & $\mathrm{~d}_{\mathrm{x}}^{2}-\mathrm{y}^{2} \rightarrow \sigma^{*}\left(\mathrm{~d}_{\mathrm{z}}^{2}\right)$ & \\
\hline & & & & 23 & $\mathrm{H}-7 \rightarrow \mathrm{L}$ & $\pi_{\mathrm{NAC}}+\sigma\left(\mathrm{d}_{\mathrm{z}}^{2}\right) \rightarrow \pi^{*}$ & \\
\hline & & & & 15 & $\mathrm{H}-1 \rightarrow \mathrm{L}+3$ & $\pi+\left(\mathrm{d}_{\mathrm{z}}^{2} / \mathrm{p}_{\mathrm{S}}\right) \rightarrow \pi^{*}$ & $(3 / 2,33)$ \\
\hline \multirow[t]{3}{*}{$\mathrm{S}_{23}$} & 3.38 & 367.2 & 0.0187 & 60 & $\mathrm{H}-7 \rightarrow \mathrm{L}$ & $\pi_{\mathrm{NAC}}+\sigma\left(\mathrm{d}_{\mathrm{z}}^{2}\right) \rightarrow \pi^{*}$ & \\
\hline & & & & 12 & $\mathrm{H}-2 \rightarrow \mathrm{L}+3$ & $\pi+\left(\mathrm{d}_{\mathrm{yz}} / \mathrm{p}_{\mathrm{S}}\right) \rightarrow \pi^{*}$ & \\
\hline & & & & 11 & $\mathrm{H}-4 \rightarrow \mathrm{L}+1$ & $\mathrm{~d}_{\mathrm{x}}^{2}-\mathrm{y}^{2} \rightarrow \sigma^{*}\left(\mathrm{~d}_{\mathrm{z}}^{2}\right)$ & \\
\hline \multirow[t]{2}{*}{$\mathrm{S}_{24}$} & 3.46 & 358.4 & 0.0051 & 49 & $\mathrm{H}-3 \rightarrow \mathrm{L}+2$ & $\mathrm{~d}_{\mathrm{xz}} \rightarrow \mathrm{d}_{\mathrm{xz}}-\mathrm{n}$ & \\
\hline & & & & 29 & $\mathrm{H}-8 \rightarrow \mathrm{L}+1$ & $\left(\mathrm{~d}_{\mathrm{yz}} / \mathrm{p}_{\mathrm{s}}\right)+\pi \rightarrow \sigma^{*}\left(\mathrm{~d}_{\mathrm{z}}^{2}\right)$ & \\
\hline $\mathrm{S}_{25}$ & 3.48 & 356.7 & 0.0028 & 83 & $\mathrm{H}-4 \rightarrow \mathrm{L}+2$ & $\mathrm{~d}_{\mathrm{x}}^{2}-\mathrm{y}^{2} \rightarrow \mathrm{d}_{\mathrm{xz}}-\mathrm{n}$ & \\
\hline \multirow[t]{2}{*}{$\mathrm{S}_{26}$} & 3.49 & 355.4 & 0.0297 & 65 & $\mathrm{H}-7 \rightarrow \mathrm{L}+1$ & $\pi_{\mathrm{NAC}}+\sigma\left(\mathrm{d}_{\mathrm{z}}^{2}\right) \rightarrow \sigma^{*}\left(\mathrm{~d}_{\mathrm{z}}^{2}\right)$ & \\
\hline & & & & 19 & $\mathrm{H}-9 \rightarrow \mathrm{L}+1$ & $\left(\mathrm{~d}_{\mathrm{z}}^{2} / \mathrm{p}_{\mathrm{S}}\right)+\pi \rightarrow \sigma^{*}\left(\mathrm{~d}_{\mathrm{z}}^{2}\right)$ & \\
\hline \multirow[t]{2}{*}{$\mathrm{S}_{27}$} & 3.59 & 345.8 & 0.0050 & 63 & $\mathrm{H}-9 \rightarrow \mathrm{L}$ & $\left(\mathrm{d}_{\mathrm{z}}^{2} / \mathrm{p}_{\mathrm{S}}\right)+\pi \rightarrow \pi^{*}$ & $\begin{array}{c}355 \\
(349)\end{array}$ \\
\hline & & & & 22 & $\mathrm{H}-8 \rightarrow \mathrm{L}$ & $\left(\mathrm{d}_{\mathrm{yz}} / \mathrm{p}_{\mathrm{S}}\right)+\pi \rightarrow \pi^{*}$ & \\
\hline $\mathrm{S}_{28}$ & 3.63 & 341.9 & 0.0004 & 95 & $\mathrm{H}-1 \rightarrow \mathrm{L}+4$ & $\pi+\left(\mathrm{d}_{\mathrm{z}}^{2} / \mathrm{p}_{\mathrm{S}}\right) \rightarrow \pi_{\mathrm{NAC}}^{*}$ & \\
\hline $\mathrm{S}_{29}$ & 3.70 & 334.7 & 0.0013 & 88 & $\mathrm{H}-4 \rightarrow \mathrm{L}+3$ & $\mathrm{~d}_{\mathrm{x}}^{2}-\mathrm{y}^{2} \rightarrow \pi^{*}$ & \\
\hline
\end{tabular}




\begin{tabular}{|c|c|c|c|c|c|c|c|}
\hline $\mathrm{S}_{30}$ & 3.71 & 333.9 & 0.0030 & $\begin{array}{l}51 \\
15\end{array}$ & $\begin{array}{l}\mathrm{H}-5 \rightarrow \mathrm{L}+2 \\
\mathrm{H}-8 \rightarrow \mathrm{L}+1\end{array}$ & $\begin{array}{l}\mathrm{p}_{\mathrm{O}(\mathrm{NAC})} \rightarrow \mathrm{d}_{\mathrm{xz}}-\mathrm{n} \\
\left(\mathrm{d}_{\mathrm{yz}} / \mathrm{p}_{\mathrm{S}}\right)+\pi \rightarrow \sigma^{*}\left(\mathrm{~d}_{\mathrm{z}}^{2}\right)\end{array}$ & \\
\hline $\mathrm{S}_{31}$ & 3.72 & 333.2 & 0.0048 & 47 & $\mathrm{H}-5 \rightarrow \mathrm{L}+2$ & $\mathrm{p}_{\mathrm{O}(\mathrm{NAC})} \rightarrow \mathrm{d}_{\mathrm{xz}}-\mathrm{n}$ & \\
\hline $\mathrm{S}_{32}$ & 3.73 & 332.2 & 0.0004 & 98 & $\mathrm{H} \rightarrow \mathrm{L}+6$ & $\mathrm{p}_{\mathrm{S}}+\mathrm{d}_{\mathrm{yz}} \rightarrow \pi_{\mathrm{Im}}^{*}$ & \\
\hline $\mathrm{S}_{33}$ & 3.76 & 330.2 & 0.0137 & $\begin{array}{l}26 \\
25\end{array}$ & $\begin{array}{l}\mathrm{H}-6 \rightarrow \mathrm{L}+2 \\
\mathrm{H}-3 \rightarrow \mathrm{L}+3\end{array}$ & $\begin{array}{l}\pi_{\mathrm{Im}} \rightarrow \mathrm{d}_{\mathrm{xz}}-\mathrm{n} \\
\mathrm{d}_{\mathrm{xz}} \rightarrow \pi^{*}\end{array}$ & \\
\hline $\mathrm{S}_{34}$ & 3.79 & 327.5 & 0.0023 & $\begin{array}{l}45 \\
33\end{array}$ & $\begin{array}{l}\mathrm{H}-1 \rightarrow \mathrm{L}+5 \\
\mathrm{H}-10 \rightarrow \mathrm{L}\end{array}$ & $\begin{array}{l}\pi+\left(\mathrm{d}_{\mathrm{z}}^{2} / \mathrm{p}_{\mathrm{s}}\right) \rightarrow \pi^{*} \\
\pi+\mathrm{d}_{\mathrm{xz}} \rightarrow \pi^{*}\end{array}$ & \\
\hline $\mathrm{S}_{35}$ & 3.80 & 326.0 & 0.0012 & $\begin{array}{l}49 \\
13\end{array}$ & $\begin{array}{l}\mathrm{H}-6 \rightarrow \mathrm{L}+2 \\
\mathrm{H}-3 \rightarrow \mathrm{L}+2\end{array}$ & $\begin{array}{l}\pi_{\mathrm{Im}} \rightarrow \mathrm{d}_{\mathrm{xz}}-\mathrm{n} \\
\mathrm{d}_{\mathrm{xz}} \rightarrow \mathrm{d}_{\mathrm{xz}}-\mathrm{n}\end{array}$ & 333 \\
\hline $\mathrm{S}_{36}$ & 3.82 & 324.2 & 0.0508 & $\begin{array}{l}23 \\
16 \\
14\end{array}$ & $\begin{array}{l}\mathrm{H}-8 \rightarrow \mathrm{L} \\
\mathrm{H}-9 \rightarrow \mathrm{L} \\
\mathrm{H}-3 \rightarrow \mathrm{L}+3\end{array}$ & $\begin{array}{l}\left(\mathrm{d}_{\mathrm{yz}} / \mathrm{p}_{\mathrm{S}}\right)+\pi \rightarrow \pi^{*} \\
\left(\mathrm{~d}_{\mathrm{z}}^{2} / \mathrm{p}_{\mathrm{S}}\right)+\pi \rightarrow \pi^{*} \\
\mathrm{~d}_{\mathrm{xz}} \rightarrow \pi^{*}\end{array}$ & $(3.72)$ \\
\hline $\mathrm{S}_{37}$ & 3.84 & 322.5 & 0.0057 & 87 & $\mathrm{H}-2 \rightarrow \mathrm{L}+4$ & $\pi+\left(\mathrm{d}_{\mathrm{yz}} / \mathrm{p}_{\mathrm{S}}\right) \rightarrow \pi_{\mathrm{NAC}}^{*}$ & \\
\hline $\mathrm{S}_{38}$ & 3.86 & 321.2 & 0.0034 & 68 & $\mathrm{H}-10 \rightarrow \mathrm{L}+1$ & $\pi+d_{x z} \rightarrow \sigma^{*}\left(d_{z}^{2}\right)$ & \\
\hline $\mathrm{S}_{39}$ & 3.96 & 313.1 & 0.0021 & 88 & $\mathrm{H}-7 \rightarrow \mathrm{L}+2$ & $\pi_{\mathrm{NAC}}+\sigma\left(\mathrm{d}_{\mathrm{z}}^{2}\right) \rightarrow \mathrm{d}_{\mathrm{xz}}-\mathrm{n}$ & \\
\hline \multirow[t]{2}{*}{$\mathrm{S}_{40}$} & 3.97 & 312.1 & 0.0034 & 72 & $\mathrm{H}-11 \rightarrow \mathrm{L}$ & $\mathrm{p}_{\mathrm{O}(\mathrm{NAC})} \rightarrow \pi^{*}$ & \\
\hline & & & & 11 & $\mathrm{H}-5 \rightarrow \mathrm{L}+3$ & $\mathrm{p}_{\mathrm{O}(\mathrm{NAC})} \rightarrow \pi^{*}$ & \\
\hline \multirow[t]{2}{*}{$\mathrm{S}_{41}$} & 3.97 & 312.0 & 0.0011 & 87 & $\mathrm{H}-5 \rightarrow \mathrm{L}+3$ & $\mathrm{p}_{\mathrm{O}(\mathrm{NAC})} \rightarrow \pi^{*}$ & \\
\hline & & & & 11 & $\mathrm{H}-11 \rightarrow \mathrm{L}$ & $\mathrm{p}_{\mathrm{O}(\mathrm{NAC})} \rightarrow \pi^{*}$ & \\
\hline $\mathrm{S}_{42}$ & 3.99 & 310.5 & 0.0005 & 99 & $\mathrm{H} \rightarrow \mathrm{L}+7$ & $\mathrm{p}_{\mathrm{S}}+\mathrm{d}_{\mathrm{yz}} \rightarrow \pi_{\mathrm{NAC}}^{*}$ & \\
\hline \multirow[t]{2}{*}{$\mathrm{S}_{43}$} & 4.01 & 308.9 & 0.0113 & 57 & $\mathrm{H}-2 \rightarrow \mathrm{L}+5$ & $\pi+\left(\mathrm{d}_{\mathrm{yz}} / \mathrm{p}_{\mathrm{s}}\right) \rightarrow \pi^{*}$ & \\
\hline & & & & 32 & $\mathrm{H}-6 \rightarrow \mathrm{L}+3$ & $\pi_{\operatorname{Im}} \rightarrow \pi^{*}$ & \\
\hline \multirow[t]{2}{*}{$\mathrm{S}_{44}$} & 4.06 & 305.4 & 0.0121 & 59 & $\mathrm{H}-6 \rightarrow \mathrm{L}+3$ & $\pi_{\operatorname{Im}} \rightarrow \pi^{*}$ & \\
\hline & & & & 22 & $\mathrm{H}-2 \rightarrow \mathrm{L}+5$ & $\pi+\left(\mathrm{d}_{\mathrm{yz}} / \mathrm{p}_{\mathrm{s}}\right) \rightarrow \pi^{*}$ & \\
\hline $\mathrm{S}_{45}$ & 4.07 & 304.7 & 0.0018 & 89 & $\mathrm{H}-11 \rightarrow \mathrm{L}+1$ & $\mathrm{p}_{\mathrm{O}(\mathrm{NAC})} \rightarrow \sigma^{*}\left(\mathrm{~d}_{\mathrm{z}}^{2}\right)$ & \\
\hline
\end{tabular}

b) Experimentally observed peaks for Glutathionylco(III)balamin (GSCbl) from A. S. Eisenberg, I. V. Likhtina, V. S. Znamenskiy, R. L. Birke, Electronic Spectroscopy and Computational Studies of Glutathionylco(III)balamin, J. Phys. Chem. A 2012, 116, 6851-6869. dx.doi.org/10.1021/jp301294x 